\title{
Developing Core Principles for Sharing Information about Potential Intervention Benefits and Harms in Patient Information Leaflets Using a Modified Delphi Survey
}

\author{
Martina Svobodova, Rachel Hale, Kerry Hood, Katie Gillies, Jennifer Bostock, Peter Bower, \\ Adrian Edwards, Sarah Rawlinson, Shaun Treweek, Jeremy Howick \\ Corresponding author: Jeremy Howick \\ Address for correspondence: HowickJ@ @ardiff.ac.uk, Centre for Trials Research (CTR), \\ College of Biomedical and Life Sciences, Cardiff University, 6th Floor, Neuadd \\ Meirionnydd, Heath Park, Cardiff, CF14 4YS
}

\section{Introduction}

The way potential harms of participating are described in a trial in participant information leaflets can sometimes cause harm. In one of our recent studies, we found that half of the participants in trials who take a placebo treatment (like a sugar pill) report having a negative 'side effect' (Howick et al. 2018). 1 in 20 of the participants who took a placebo dropped out due to such a 'side effect.' This could be due to misattribution (whereby a symptom that would have arisen whether or not the patient was in the trial is attributed to the trial treatment), as well as negative expectations or 'nocebo effects.' A trial participant might be warned about a possible side effect in a way that caused them to expect, and then actually experience, this side effect. Negative side effects among participants taking placebos were more common in pain-related, cancer, and mental health trials. However, our research has also shown that harms can be presented in a way that is honest, balanced, avoids coercion and at the same time does not induce unnecessary communication-induced harms.

In another study, we looked at 33 Participant Information Leaflets (PILs) used for National Institute of Health Research (NIHR) trials in the UK, and found that the way information about harms was shared did not seem to follow any logical pattern (Kirby et al. 2020a). Most of them had more information about harms than potential benefits, and some did not mention potential benefits at all. This can cause information-induced adverse events ('nocebo effects').

Because guidance regarding the best way to present potential trial participation benefits and harms does not exist, every principal investigator has to negotiate their own method for sharing information about trial benefits and harms. This is reinventing the wheel and wastes scarce resources.

\section{Aim of the Study}

The aim of this study is to understand what information about trial harms and benefits stakeholders consider to be important for 'principled participant information leaflets' or 'PrinciPILs' to contain. The stakeholders will include patients, ethics committee members, industry representatives, medico-legal experts, psychologists, and trial managers. 


\section{Primary Outcome}

List of key principles to be considered when sharing information about potential trial benefits and harms within PILs.

\section{Study Design}

We are using a modified Delphi survey, and have appealed to the Guidance on Conducting and Reporting Delphi Studies (CREDES) to guide the reporting of this protocol (Junger et al. 2017). The Delphi method is recommended for developing guidance, the modified Delphi method (which includes an expert meeting at the end) can be superior for maximising cooperation between interdisciplinary researchers (Graefe and Armstrong 2016), and has been used successfully in similar areas (Gillies et al. 2015; Howick et al. 2020).

\section{Development of the List of Items for the Delphi}

We will generate a list of potential information about benefits and harms from three sources that our background research has revealed to be important (Howick et al. 2018; Kirby et al. 2020b; Howick 2020).

a. principles and examples from our review of UK PILs; (Kirby et al. 2020b)

b. extracted principles and example from a random sample of Drug Facts Boxes; (Schwartz, Woloshin, and Welch 2009)

c. statements in official guidance about presenting trial benefits and harms in PILs from within the UK (e.g. HRA: https://bit.ly/303HGg5) and internationally (e.g. European Medicines Agency (EMA): https://bit.ly/2MqCPO2; World Health Organisation (WHO): https://bit.ly/2U6mx12; The United States Food and Drug Administration (USFDA): https://bit.ly/3dvMe2K).

These will be collated, and the list will be piloted for face validity by our patient and public representatives. The information sources will then be used to generate a comprehensive list of principles about benefits and harms. The co-applicants, supplemented by the patient and public involvement (PPI) representative and advisory group will remove redundancy.

\section{Sample Size}

There is currently no standard method for determining sample size calculations for Delphi studies and the planned sample size is not derived statistically (Gargon et al. 2019). While 5 to 10 experts per expert group are considered adequate for content validation, we aim to sample 20 people per stakeholder group. This number is based on a conservative estimate of a $50 \%$ dropout rate between Delphi rounds, and a study by Harman et al. who used a lower limit of 10 in any stakeholder group (Harman et al. 2013). Other studies also suggest a minimum number of panel members ranging from 10 to 20 panel members per area of expertise (Okoli and Pawlowski 2004; Slade et al. 2014).

Efforts will be made to maximise response across stakeholder groups and to achieve diverse representation. We will use our collective networks to promote balance in terms of gender, age, and ethnic background. We will also record this data in the Delphi survey. The total number of respondents per each stakeholder group will be reviewed in between rounds. 


\section{Participant Identification}

A group of stakeholders will be identified from the contact lists and networks of coapplicants and PPI representatives. The survey will only be available in English language due to the time-limited nature of the study. Consideration has been given to the representativeness of the stakeholders that will be included and the ability of people across the different groups to engage with the consensus process. Stakeholders will include representatives from each of the seven following sources:

1. Public, patients and their advocates. Our PPI representative (Jennifer Bostock) and advisory board member (Jono Broad) will help us identify these from their networks including PainUK and People in Research, National Institute for Health Research (NIHR), the James Lind Alliance, the NIHR centre for Engagement and Dissemination and from health literacy groups (such as, www.healthliteracy.org.uk). They will also be selected based on experience with one of the conditions in which information induced harm is more likely (cancer, musculoskeletal conditions and mental and behavioural disorders).

2. Ethics committee members and other approvals staff. The PI and co-applicants have contacts at the Health Research Agency (HRA) who have expressed willingness to put us in touch with interested ethics committee members and chairs.

3. Industry (including medico-legal experts). We will identify these with help from our industry partners (including the Association of British Pharmaceutical Industry) and with input from the advisory board of medico-legal experts.

4. Applied Researchers, including psychologists and risk communicators. These will be identified by the PI and co-applicants, who have an extensive network of psychologists with relevant expertise. We will target psychologists with a range of relevant expertise, especially in risk communication and behavioural science.

5. Research Nurses, Clinical trial managers and trialists. We will identify these via the UK Clinical Research Collaboration (UKCRC) Registered Centre for Trials Research at Cardiff University and the UK Trial Managers' Network (UKTMN).

\section{Delphi Survey: Design}

We will use Qualtrics for the Delphi survey. Participants will be invited to participate by email asking them to complete the online Delphi questionnaire through a web link embedded in the email. The process will be conducted anonymously in order to reduce the risk of any single respondent's potential biases dominating the process or conclusions.

Following methods used in a related study (Gillies et al. 2015), our Delphi survey will begin with a brief introduction of the aim of the study, how the information collected will be used and stored, how the findings will be made available to participants, and will remind participants of the importance of competing all rounds. Participants will be asked to tick a box at the start of the survey to confirm that they agree to participate and that they understand that they have a right to withdraw from the study at any time. Participants who do not confirm will be considered as having withdrawn from further participation. Reminder emails will be sent to non-responders at each round.

\section{Data management}

Survey data will be entered/stored/retrieved in Medidata Rave; a fully validated Electronic Records Electronic Signatures (ERES) / GCP compliant, secure, web-based interface 
electronic data capture system. At the end of the study, data will be anonymised, IP addresses and location data will be removed and responses will be disconnected from the participants who provided them.

\section{Figure 1. Study Schema}

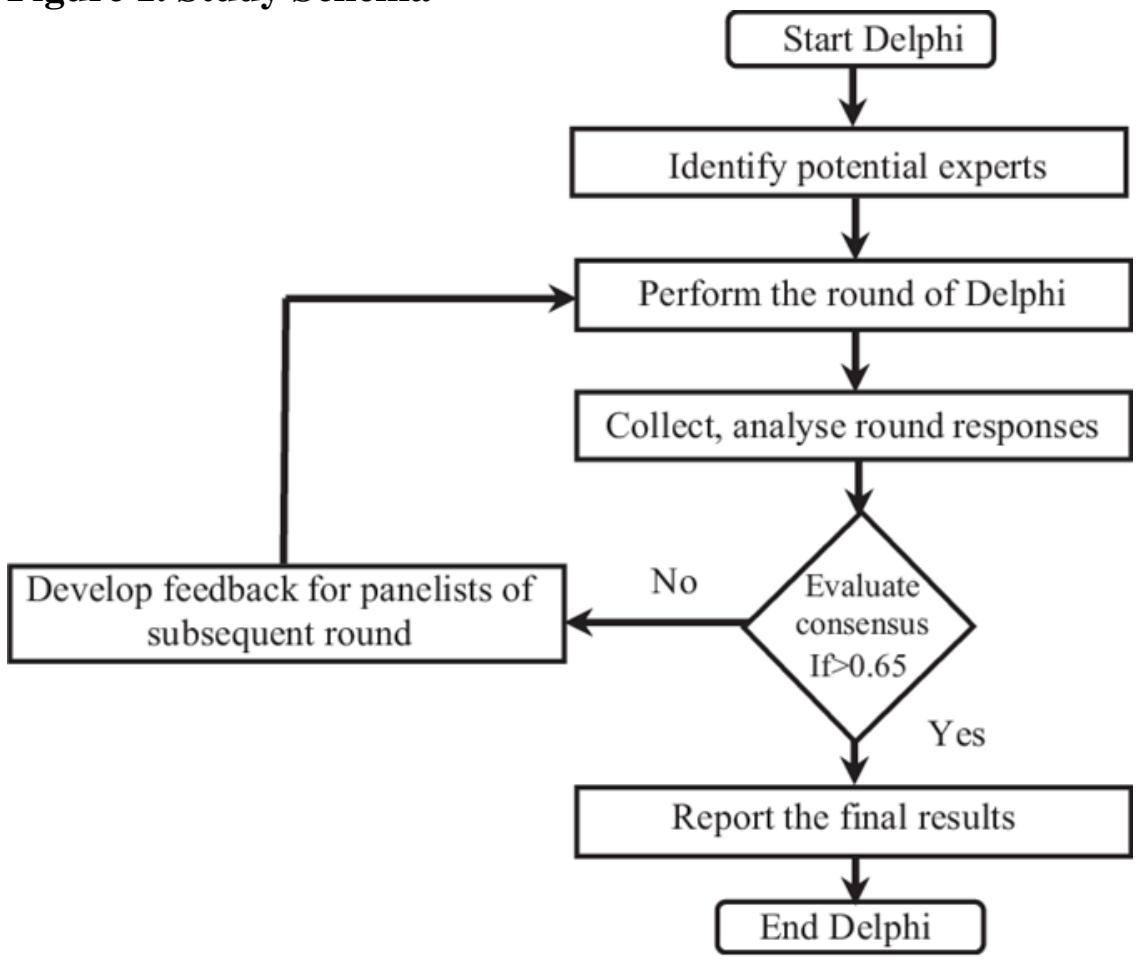

\section{Figure 2: Participant Flow Diagram}

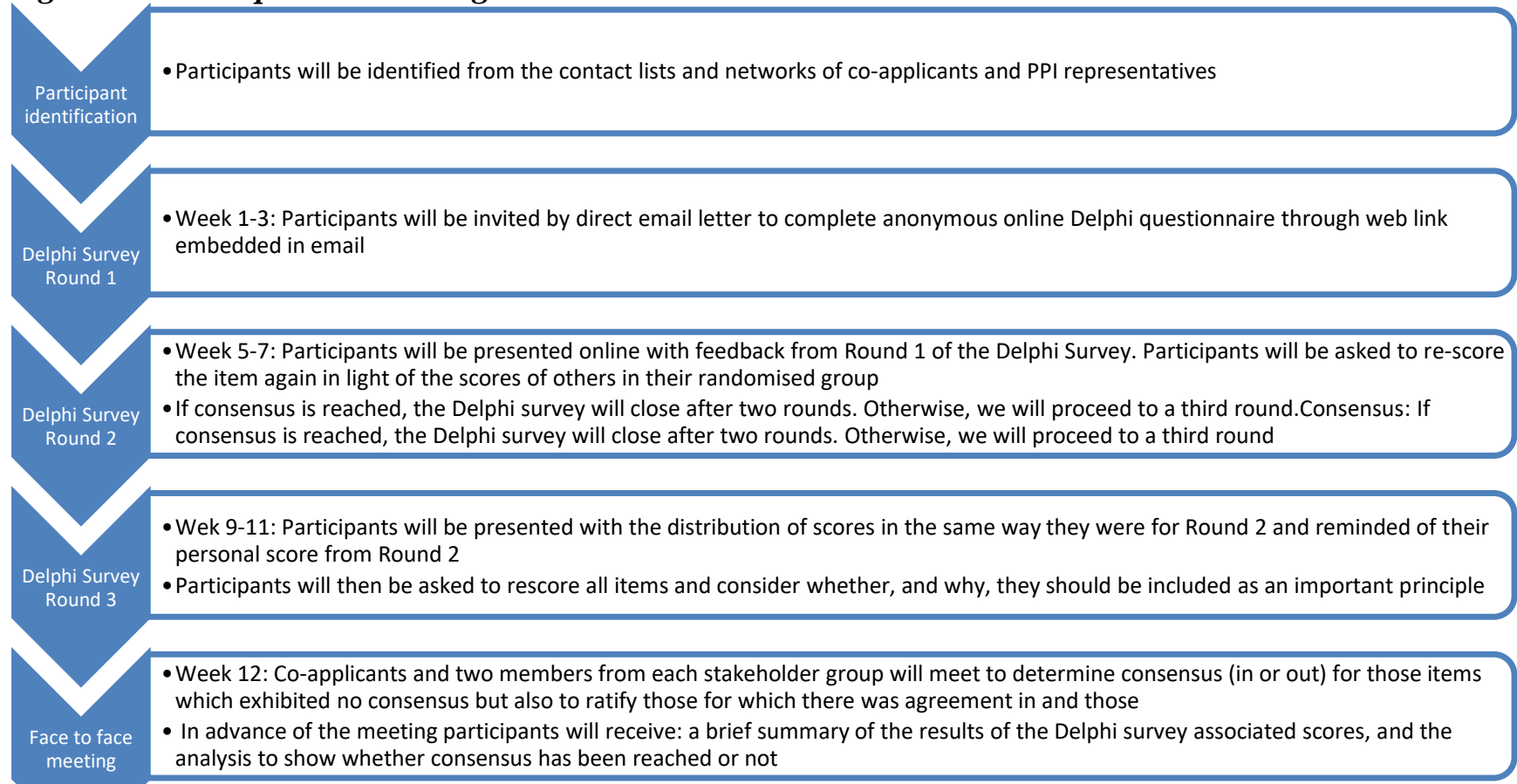




\section{Delphi Survey: Conduct}

The study schema (Figure 1) shows how the Delphi survey process will be conducted. The participant flow diagram (Figure 2) outlines the progress of the Delphi survey participants through each stage of the Delphi process.

\section{Delphi Round 1}

The survey will be presented in an online format (see Appendices 1 and 2). Each participant will get a personalized trackable link that can only be used once. Participant's email will be automatically saved with their survey data. This will allow identification of individuals as they progress through the Delphi process, tracking responses in progress and also sending out reminders and thank you messages. In addition, this link will automatically save the participants' data as they progress through the survey. Each respondent will be asked to identify their stakeholder group (patient and advocates, research ethics committee member, and so forth).

\section{Survey Format}

Participants will be asked to rate their agreement or disagreement with 27 statements using the scale of 1 to 9 . For purposes of analysis, we will divide these into clusters of 3 representing agreement, indecision, and disagreement.. Participants will also be given the opportunity to share free text comments where they can provide reasons for their answers and suggest other features they believe are important.

We acknowledge that the principles are relative to the severity of the potential harms as well as the magnitude of the potential benefits. Therefore, we will use vignettes (based on real examples) to ask participants whether the way benefits and harms are presented should be different across a variety of magnitudes of potential trial harms and potential trial benefits.

\section{Round 1 Analysis}

Descriptive statistics will be used to summarise the results from each round. For each item, the distribution of scores (frequency distribution, the median and interquartile range) will be summarised by stakeholder group alongside the total number of participants who scored the item. Any new additional items listed by participants in round 1 will be reviewed and coded by two members of the study team to ensure they are independent from those listed, with a third reviewer acting as an arbiter if there is disagreement. Participants will be instructed to rate all items on their own merit even if they appear similar. All items will be carried forward to round 2.

\section{Response Rate in Round 1}

Following the completion of round 1, the research team will tabulate an overview of the total number of registrations to the website, the number of participants who have completed round 1 , the total number of participants in each stakeholder group and the number of respondents as a percentage of those invited by stakeholder group.

Round 2 assumes sufficient numbers respond to round 1 across each of the stakeholder groups. If there are inadequate numbers for one or more stakeholder groups, the study 
advisory group will be consulted. Those who have not taken part in round 1 will not be invited to participate in round 2.

\section{Delphi Round 2}

Round 2 of the Delphi survey will also be presented online. Participants will be presented with feedback (number of respondents, distribution of scores across the nine-point scale for their particular stakeholder group, and collated free text comments). This will also include information on any modifications of the survey instrument such as deletion, addition or modification of survey items based on the previous round. Participants will be shown their score from round 1 and will be asked to re-score the item again in light of the scores of others in their randomised group, using the same nine-point scale.

\section{Round 2 Analysis}

As in round 1, descriptive statistics will be used to summarise the results and for each item, the distribution of scores will be summarised across stakeholder groups. If consensus is reached (see 'Definition of consensus' below), the Delphi survey will close after two rounds. Otherwise, we will proceed to a third round.

\section{Response Rate in Round 2}

The total number of participants invited to participate in round 2 will be recorded and compared to the total number of round 1 responders.

\section{Delphi Round 3}

If stability is not reached after two rounds, we will conduct a third round of the survey. In the final online round of the Delphi, participants will be presented with the distribution of scores in the same way they were for round 2 and reminded of their personal score from round 2. Participants will then be asked to rescore all items. The total number of participants invited to round 3 will be recorded and compared to the total number of round 2 responders.

\section{Round 3 Analysis}

For the final analysis, for each item, the number of participants who scored the item and the distribution of scores will be summarised, alongside the number of respondents who scored the items across all three rounds. The proportion of respondents scoring 1 to 4,5 and 6 to 9 on the scale will be calculated for each item. Each item will be classified as: 'consensus in', 'consensus out' or 'no consensus' according to the study's definition of consensus. We will analyse the open-ended responses by grouping and summarizing similar answers, using response frequencies to assess the closed-ended responses.

\section{Face to Face Meeting}

For the final step of this modified Delphi method, we will convene a face-to-face (if possible) meeting with the co-applicants, and two members from each stakeholder group. The main aim of the meeting will be to determine consensus (in or out) for those items which exhibited no consensus but also to ratify those items for which there was agreement. 
In advance of the meeting participants will receive a brief summary of the results from each round of the Delphi survey, and the analysis to show whether consensus has been reached or not.

The discussion will be facilitated by the study team. The format and process of the consensus meeting will take account of the potential issues of power and communication and other barriers that may affect participation by some stakeholders. Strategies will be used to minimize the influence of power differentials between different stakeholders during the meeting and provide support for their inclusion, including ensuring good preparation, an enabling environment with strong support for participants, and the use of good facilitation skills.

The items that achieved consensus will be presented briefly and participants will be asked to voice any disagreement. The focus for the rest of the meeting will be on the items with no consensus. Each statement will be presented in turn alongside its scores from individual stakeholder group Delphi responses. Discussion will be welcomed requesting any points of clarification and an opportunity to consider whether the item should be considered as important. At this stage it will also be important to consider whether any of the items being considered are surrogates for any items already listed within those for which there was consensus (in or out).

Participants will then be asked to vote (using polleverywhere.com) on whether the statement is important or not. The same voting scale used in the Delphi will be applied. For an item to be voted in more than $70 \%$ of the group have to agree on its inclusion i.e. rate it 1-4. If less than $70 \%$ score it 1-4 it will not be included. At the end of the voting process the final results set will be presented to the group.

\section{Definition and Attainment of Consensus}

We will take consensus to be defined as follows:

- Consensus in (that an item is important): agreement of a substantial majority $(\geq 70 \%)$ of panellists that an item is of critical importance.

- Consensus out (that an item is not important): agreement of a substantial majority $(\geq 70 \%)$ of panellists that an item is of limited importance.

- No consensus: Anything else.

The cut-off reflects recommended quality indicators for a Delphi study (Diamond et al. 2014). Items about which there is no consensus will be discussed in the face-to-face meeting, with an aim to resolving them or ensuring how to consider the lack of consensus in the eventual PrinciPILs.

We will explore reasons for stable non-consensus and take these into account in our PrinciPIL development. For example, if there is disagreement about whether information about benefits should come before information about harms, we might consider using both methods. We will document the discussions during the consensus meeting paying particular attention to any gaps or minority concerns about the list that achieved consensus so as to reflect on any potential limitations. These discussions will also provide further opportunity to hear a refined discussion of the reasoning for regarding some item domains as important. We anticipate that there may not be consensus that there is a 'one size fits all' approach to sharing information about potential harms. The way in which information about potential harms is shared, is likely to depend on a number of factors including the degree of potential harm (mild versus serious) and the degree of the potential benefit (minimal, moderate, or 
large). It may nevertheless be possible to stratify the communication methods depending on the degree of risk and benefit.

\section{Discussion}

Patient representatives and other stakeholders are already involved in designing PILs for patients in trials. However, there is currently no guidance specifically on how to share information about potential intervention benefits and harms. In this study, we will start the process of overcoming this gap.

\section{Ethical and Governance Approval}

Ethical approval has been obtained from Cardiff University School of Medicine Research Ethics Committee prior to commencing the survey (SMREC reference 21/54).

\section{Funding}

This study is funded by the Medical Research Council. 


\section{Appendix 1: Participant Information Sheet and Study Consent Form to participate in a research study}

Title of Research study: The PrinciPILs Study: Developing and Testing Participant Information Leaflets (PILs) that Inform and do not Cause Harm (PrinciPILs)

Chief Investigator: Dr Jeremy Howick

Study Coordination: Centre for Trials Research, Cardiff University, 6th Floor, Neuadd Meirionnydd, University Hospital of Wales, Heath Park, Cardiff CF14 4YS Thank you for your interest in the PrinciPIL study!

\section{Why are we doing this study?}

A clinical trial is a research study conducted in human beings that tries to find out whether treatments benefit patients and are safe. Anyone who is thinking about taking part in a clinical trial needs to be told about the potential benefits and harms of taking part. At the moment, the way potential harms and benefits are shared varies a lot, depending on the trial. Sometimes, information about possible benefits and harms is provided, and at other times, possible benefits are not mentioned at all.

Meanwhile, research shows that telling people about harms in certain ways can cause harm. For example, some studies have shown that if a patient is told a placebo pill (like a sugar pill) might cause mild headaches, then that patient may report they had a headache. The headache was not caused by the placebo pill, but by the doctor telling the patient they might get a headache: this is called a "nocebo effect."

We would like to find a consistent way to tell potential clinical trial participants about possible harms which is honest, balanced but minimizes "nocebo effect" harms.

This survey is the first step in this study. We will ask members of the public, nurses, doctors, ethicists, lawyers, and clinical trial managers what they think about how to present information about possible clinical trial benefits and harms to participants.

The survey includes statements on how information on possible benefits and harms in a clinical trial should be shared with a potential trial participant when they are considering whether to participate. You will be asked whether you agree or disagree with each statement.

\section{What is involved?}

There will be two rounds of this survey. For our results to be trustworthy, we would really appreciate it if you completed both rounds. Each round takes about 15 minutes to complete. They will be online and will be completely anonymous. We would like you to complete this first round of the survey by DD/MM/2021. After we've received responses to this first round, we will summarise the results to help prepare for the second round of the survey. We will contact you when the second round is ready for you to complete.

Taking part is completely voluntary and you are free to withdraw from the study at any time without giving a reason. If you need help completing the survey you can ask those around you (friends or family members) to help.

In order for us to track how many people have completed the survey, and to invite you to the second round, we would like you to initially register using your email address. When you register, you will be given a study number which will be used to process your data anonymously. Nobody will be able to track your individual answers to you (see below for further information). 


\section{How will I benefit from taking part in this study?}

Although there are no direct health benefits to you, this study is part of a project that will benefit people who participate in clinical trials in the future. By developing a better way to share information about treatment benefits and harms, potential trial participants will probably experience less harm, and even be more willing to participate in research. It will therefore improve trial participants' quality of life, and at the same time improve health research, which ultimately benefits all of us.

We also hope our findings will eventually be useful outside trials, in clinical practice where nurses and doctors need to tell their patients about potential harms and benefits of treatments.

\section{Will my part in the study be confidential?}

Yes. All your data will be kept safe and secure in accordance with the General Data Protection Regulation (GDPR) and all information about you will be handled in confidence.

Your email contact details will be used strictly for the purpose of completing this study and will not be shared with anyone unless absolutely necessary for the purpose of this study.

Cardiff University will be the data controller for this study. Cardiff University is responsible for looking after your information and using it properly. Study data will be stored securely and only authorised staff on the study team or from regulatory authorities, who check the quality of the study, will be given access to the data. The people who analyse the information will not have access to your contact details.

At the end of the study, we will keep some of the anonymised data at an approved storage facility for a minimum of 15 years in case we need to check it, or for future research. This data will be completely anonymised, so nobody will ever be able to identify respondents.

Cardiff University data protection information can be found here.

\section{Where can I find more information about this study?}

A full version of the protocol, which includes a description of how the statements for this survey were identified, can be found here.

If you have any questions or at any time you would like to discuss this survey, please contact the PrinciPIL team at Cardiff University principil@ cardiff.ac.uk or 02920687463.

Thank you for reading this and participating in our study!

Register $\square$ By completing this questionnaire, I am consenting to take part in this study. I understand my data will be held anonymously and securely and that I have a right to withdraw from this study at any time by contacting a member of the study team. I understand that when this information is no longer required for this purpose, official University procedure will be followed to dispose of all data.

If you have forgotten your login details click here 


\section{Appendix 2: Delphi Survey Statements (to be presented online via Qualtrics)}

Instructions: Please rate how much you personally agree or disagree with the following statements. Some of the statements are introduced with a scenario. Please read the scenario carefully before rating the relevant statement.

Throughout the survey we will use the following scale:

1. Strongly agree

2. Agree

3. Moderately agree

4. Mildly agree

5. Undecided

6. Mildly disagree

7. Moderately disagree

8. Disagree

9. Strongly disagree

\section{Section 1: Scenarios}

\section{Scenario 1. Giving a participant too much information about possible harms}

John was thinking about enrolling in a trial of a new drug to treat migraines. He read the participant information leaflet very carefully, and in several places the leaflet contained information about gastrointestinal side effects. He didn't know what this meant so he looked it up and figured out that it probably meant stomach aches and nausea. He didn't find much information about the benefits of the new drug, but his doctor said it was worth a try and why would his doctor say that if it wasn't going to help? John enrolled in the trial, and his migraine symptoms got a bit better, but every evening he suffered from stomach aches and withdrew from the trial.

1. Potential harms that are not very serious do not need to be emphasized

\section{Scenario 2. Not telling a participant about harms enough}

Jane had enrolled in a trial of a new 'disease modifying antirheumatic drug' (DMARD) to treat arthritis. The drug seemed to work very well. Then, she read an article in the newspaper about how DMARDs can increase the risk of getting some types of cancer. She asked the trial doctor, who said that the risk of cancer was very low. He then showed her the participant information leaflet she had signed a number of months ago. It listed cancer as a possible side effect only towards the end of the document, using language Jane didn't understand and thought was the 'small print.'

2. Potentially serious harms need to be emphasized, even if they are very rare

\section{Scenario 3. Comparison with what happens if we take nothing}

Marie stopped taking her statin drugs because they caused muscle pain. Her doctor suggested that she should try alternating between taking statins and taking a placebo pill every two weeks for three months. She didn't know whether she was taking the statin or the placebo. At the end of the three months, her doctor showed her that she had as much muscle pain with the placebo as she did with the statin.

3. Potential benefits and harms of a clinical trial need to be compared with what happens if the participant does not take part in the trial 


\section{Scenario 4. Positive framing}

Two trials were recruiting people with psoriasis to test new drugs. Andrew had psoriasis and asked about information about both trials. In the information leaflet describing the first trial, the drug was described as having 'common side-effects, affecting 1 in 10 people'. In the second, the side-effects were described as 'uncommon: $90 \%$ of people will not be affected.' Andrew felt safer enrolling in the second trial.

Saying (1) 'this drug has a common side effect that affects 1 in 10 people' is logically the same thing as saying (2) 'this drug has an uncommon side effect: $90 \%$ of people who take it are not affected.' The second way of saying it is called 'positive framing'

4. It is okay to use 'positive framing' when describing how severe harms can be.

\section{Section 2: Describing potential benefits of a clinical trial}

5. Benefits are never completely certain, so they should not be described

6. Potential benefits should be described more fully than potential harms

7. The most likely potential benefits should be described

8. Any likely benefits to the participant (including embryos, foetus, nursing infants) should be described

9. General potential benefits (such as 'the medicine may help you and your cancer') should be described

10. Concrete, specific potential benefits (such as 'this medicine is designed to enable you to walk farther before becoming breathless') should be described

11. Only the most important potential benefits should be described. If too many are included the reader might become confused. A complete list can be contained in an appendix or online.

\section{Section 3: Describing potential harms of a clinical trial}

12. Participants should not be told about potential harms

13. Potential harms should be described more fully than potential trial benefits

14. Only the most common possible harms should be mentioned. This will focus the reader's attention and minimize overload

15. The harms should be separated into serious (life threatening, causing permanent damage) and less serious (like a mild headache that goes away quickly)

16. Not all potential harms are known, especially for new treatments that have not been studied extensively. Participants need to know that not all potential harms can be listed.

17. Sometimes harms are discovered after the trial begins. As soon as they are discovered, participants need to be told about them

18. Risks to conceiving/fathering a child, pregnancy, or breastfeeding should be emphasized

19. It's okay to use 'positive framing'. That is, it is okay to say 'this treatment is safe for $90 \%$ of the people who take it' instead of 'this treatment causes side effects for $10 \%$ of the people who take it'

20. Potential harms should be described in pictures as well as words

21. Potential trial harms should be described in such a way that they can be compared to what would happen if participant did not take part in the trial

\section{Section 4: The ordering and placement of benefits and harms in the participant leaflet layout}

22. Potential benefits should be described after harms

23. Potential benefits and harms should be beside to each other (for example in two columns)

24. Information about potential benefits or harms should be presented apart by one or more pages 
25. Information about potential benefits and harms should be mentioned in more than one place in the leaflet.

26. A complete (detailed) description of the potential harms (and the likelihood of each harm) should be provided in a table in an appendix

27. Drug fact boxes (see below) divide harms into serious and non-serious. This way of presenting harms is helpful.

\begin{tabular}{|c|c|c|c|}
\hline What difference did ABILIFY make? & $\begin{array}{l}\text { Anti-depressant } \\
+ \text { ABILIFY } \\
\text { (10 mg each day) }\end{array}$ & vs. & $\begin{array}{l}\text { Anti-depressant } \\
+ \text { PLACEBO } \\
\text { (No drug) }\end{array}$ \\
\hline $\begin{array}{l}\text { How dld ABILIFY help? } \\
\text { Depression scores improved by } 3 \text { points more than placebo } \\
\text { (on a scale from } 0 \text { to } 60 \text { ). }\end{array}$ & 9 points better & vs. & 6 points better \\
\hline $\begin{array}{l}11 \% \text { more people had an important response and were no longer } \\
\text { considered to have major depression }\end{array}$ & $26 \%$ & vs. & $15 \%$ \\
\hline $\begin{array}{l}\text { Functioning scores improved by } 0.5 \text { points more than placebo } \\
\text { (on a scale from } 0 \text { to } 10 \text { ). }\end{array}$ & 1.2 points better & vs. & 0.7 points better \\
\hline \multicolumn{4}{|l|}{ What were ABILIFY'S side effects? } \\
\hline $\begin{array}{l}\text { Serlous slde effects } \\
21 \% \text { more people developed akathisia - severe restlessness that makes it } \\
\text { hard to keep still }\end{array}$ & $25 \%$ & vs. & $4 \%$ \\
\hline $3 \%$ more people developed movement disorders -like Parkinson's disease & $8 \%$ & vs. & $5 \%$ \\
\hline \multicolumn{4}{|l|}{ Symptom side effects } \\
\hline $5 \%$ more had blurred vision & $6 \%$ & vs. & $1 \%$ \\
\hline $4 \%$ more had substantial weight gain & $5 \%$ & vs. & $1 \%$ \\
\hline $4 \%$ more had fatigue & $8 \%$ & vs. & $4 \%$ \\
\hline $3 \%$ more had constipation & $5 \%$ & vs. & $2 \%$ \\
\hline
\end{tabular}

WARNINGS ABOUT UNCONMON LIFE-THREATENING AND VERY SERIOUS SIDE EFFECTS

Young adults using anti-depressants for major depression have a higher risk of suicidal thinking and behavior. Elderly patients with dementia-related psychosis should not use antipsychotic drugs - Ilke ABILIFY-because they increase death. Antipsychotic drugs cause: Neuroleptic Malignant Syndrome (very high fever and blood pressure, delirium), Tardive Dyskinesia (uncontrollable facial / body movements), Dangerous Heart Rhythms, Seizures, Low White Blood Cells, Trouble Swallowing, Aspiration Pneumonia, Diabetes, Low Blood Pressure, Trouble Regulating Body Temperature 


\section{References:}

Diamond, I. R., R. C. Grant, B. M. Feldman, P. B. Pencharz, S. C. Ling, A. M. Moore, and P. W. Wales. 2014. 'Defining consensus: a systematic review recommends methodologic criteria for reporting of Delphi studies', J Clin Epidemiol, 67: 401-9.

Gargon, E., R. Crew, G. Burnside, and P. R. Williamson. 2019. 'Higher number of items associated with significantly lower response rates in COS Delphi surveys', J Clin Epidemiol, 108: 110-20.

Gillies, K., V. Entwistle, S. P. Treweek, C. Fraser, P. R. Williamson, and M. K. Campbell. 2015.

'Evaluation of interventions for informed consent for randomised controlled trials (ELICIT): protocol for a systematic review of the literature and identification of a core outcome set using a Delphi survey', Trials, 16: 484.

Graefe, A., and J.S. Armstrong. 2016. 'Comparing face-to-face meetings, nominal groups, delphi and prediction markets on an estimation task', Int J Forecasting, 27: 183-95.

Harman, N. L., I. A. Bruce, P. Callery, S. Tierney, M. O. Sharif, K. O'Brien, and P. R. Williamson. 2013. 'MOMENT--Management of Otitis Media with Effusion in Cleft Palate: protocol for a systematic review of the literature and identification of a core outcome set using a Delphi survey', Trials, 14: 70.

Howick, J. 2020. 'Unethical informed consent caused by overlooking poorly measured nocebo effects', J Med Ethics.

Howick, J., R. K. Webster, J. L. Rees, R. Turner, H. Macdonald, A. Price, A. W. M. Evers, F. Bishop, G. S. Collins, K. Bokelmann, S. Hopewell, A. Knottnerus, S. Lamb, C. Madigan, V. Napadow, A. N. Papanikitas, and T. Hoffmann. 2020. 'TIDieR-Placebo: A guide and checklist for reporting placebo and sham controls', PLoS Med, 17: e1003294.

Howick, J., R. Webster, N. Kirby, and K. Hood. 2018. 'Rapid overview of systematic reviews of nocebo effects reported by patients taking placebos in clinical trials', Trials, 19: 674.

Junger, S., S. A. Payne, J. Brine, L. Radbruch, and S. G. Brearley. 2017. 'Guidance on Conducting and REporting DElphi Studies (CREDES) in palliative care: Recommendations based on a methodological systematic review', Palliat Med, 31: 684-706.

Kirby, Nigel, Victoria Shepherd, Jeremey Howick, Sophie Betteridge, and Kerenza Hood. 2020a. 'Nocebo effects and participant information leaflets: evaluating information provided on adverse effects in UK clinical trials', Trials, 21: 658.

Kirby, Nigel, Victoria Shepherd, Jeremy Howick, Sophie Betteridge, and Kerenza Hood. 2020b. 'Nocebo effects and participant information leaflets: evaluating information provided on adverse effects in UK clinical trials', forthcoming.

Okoli, Chitu, and Suzanne D. Pawlowski. 2004. 'The Delphi method as a research tool: an example, design considerations and applications', Information \& Management, 42: 15-29.

Schwartz, L. M., S. Woloshin, and H. G. Welch. 2009. 'Using a drug facts box to communicate drug benefits and harms: two randomized trials', Ann Intern Med, 150: 516-27.

Slade, Susan C, Clermont E Dionne, Martin Underwood, and Rachelle Buchbinder. 2014. 'Standardised method for reporting exercise programmes: protocol for a modified Delphi study', BMJ Open, 4: e006682. 